\title{
A Phase Difference Measurement Method Based on the Extended Kalman Filter for Coriolis Mass Flowmeters
}

\author{
Nan Chen ${ }^{1,2}$, Shangchun Fan ${ }^{1}$, Dezhi Zheng ${ }^{1}$ \\ ${ }^{1}$ School of Instrumentation and Optoelectronics Engineering, Beihang University, Beijing 100191, China, \\ zhengdezhi@buaa.edu.cn \\ ${ }^{2}$ School of Mechanical and Electrical Engineering, Beijing Polytechnic, Beijing 100176, China
}

\begin{abstract}
According to the characteristics of stable single-phase flow, a phase difference measurement method based on the extended Kalman filter is proposed in this paper for use with Coriolis mass flowmeters. Firstly, the Mallat algorithm is applied to filter out interference signals. Then, the frequency and phase difference of the two reconstructed signals are detected through the extended Kalman filter. Compared with the sliding Goertzel algorithm or discrete time Fourier transform, the proposed method does not need to predict the signal frequency and avoids quadratic error. Simulations and experiments show that the proposed method has stronger anti-interference, higher measurement accuracy and lower relative error than the existing method based on the Hilbert transformation.
\end{abstract}

Keywords: Coriolis mass flowmeter, phase difference, extended Kalman filter, Mallat algorithm.

\section{INTRODUCTION}

Coriolis mass flowmeters $(\mathrm{CMF})$ can directly measure fluid mass flowrates with high precision and have many applications in various industries. The mass flowrate is obtained by measuring the phase difference between two vibration signals of the same frequency that are detected by electromagnetic sensors [1], [2]. Therefore, accurate phase difference estimation is essential for CMF.

At present, the main methods for CMF phase difference estimation include discrete Fourier transform (DFT) [3]-[6], sliding Goertzel algorithm (SGA) [7]-[9], discrete time Fourier transform (DTFT) with negative frequency contribution [10], and Hilbert transform (HT) [11]-[13]. The DFT can effectively suppress the interference of harmonics and random noise, and has good anti-interference ability. However, due to non-periodic sampling, spectral leakage can result in reduced accuracy of phase difference calculations. The SGA eliminates the spectrum leakage caused by nonperiodic sampling of DFT, but there is a slow convergence rate and a numerical overflow problem in practical applications. DTFT with negative frequency contribution has a higher accuracy than traditional DTFT algorithms, but the iterative process makes for a large computational load. Both SGA and DTFT algorithms need to know the frequency in advance to calculate the phase difference. The HT method is computationally efficient and can obtain the phase difference without information on signal frequency [14]. However, its anti-interference performance is poor and it needs a strict prefilter. Singular value decomposition (SVD) [14] and polyphase decimation filtering and band-pass filtering [13] have been applied to $\mathrm{CMF}$ to filter out noises.

A phase difference measurement method based on the extended Kalman filter (EKF) for CMF is proposed in this paper. It eliminates the need to know the frequency when calculating the phase difference and provides more precise estimates than the existing methods. In Section 2, the process of the new method is presented, including noise reduction, state space description, and EKF. The performance of the proposed method is validated by simulations and experiments in Section 3. Conclusions are drawn in Section 4.

\section{PRINCIPLE OF PHASE DIFFERENCE MEASUREMENT BY EKF}

\section{A. Noise reduction based on the Mallat algorithm}

The Mallat algorithm [15] is a fast algorithm used for wavelet analysis. It is a tower algorithm based on multiresolution scenarios, and was proposed by Mallat for use with discrete signals. The difficulty of calculating wavelet coefficients is greatly reduced with the Mallat algorithm. The essential process of the Mallat algorithm is to obtain two sets of parameters: 1) approximate coefficients from a low-pass filter, and 2) detailed coefficients from a high-pass filter. Then, in the same way, the low frequency part is taken as the input signal and decomposed again. This kind of signal decomposition can decompose a mixed signal composed of different frequencies into sub-signals of different frequency bands. It can be effectively used for signal feature extraction, signal and noise separation, signal analysis, and reconstruction. 
According to the Mallat algorithm process, the appropriate wavelet function should be selected. The signal is decomposed layer by layer to obtain the wavelet coefficients of each layer. Then, according to a certain algorithm, the wavelet coefficients of each layer are processed to eliminate noise from the signal. After reconstruction, the final wavelet coefficient matrix of the target signal retains only the valid information.

The output signal $f(t)$ of a CMF sensor can be expressed by the following equation:

$$
f(t)=f_{i}(t)+f_{s}(t)
$$

where $f_{i}(t)$ is the effective signal; and $f_{\mathrm{s}}(t)$ is signal noise, including harmonics and random noise.

Finite layer decomposition of the signal is performed using the Mallat algorithm:

$$
\begin{aligned}
& f(t)=f_{J}^{A}(t)+\sum_{j=1}^{J} f_{j}^{D}(t)=A_{J}+\sum_{j=1}^{J} D_{j}=A_{1}+D_{1} \\
& =A_{2}+D_{2}+D_{1}=\cdots
\end{aligned}
$$

where $\mathrm{J}$ is the number of decomposition layers; $A_{J}=f_{J}^{A}(t)$ represents the approximate coefficients; and $D_{J}=f_{j}^{D}(t), j=1,2, \cdots J \quad$ represents the detailed coefficients.

In this paper, the Daubechies wavelet is used to decompose the CMF output signal in five layers:

$$
f(t)=A_{5}+D_{4}+D_{3}+D_{2}+D_{1}
$$

When the signal is reconstructed, the detailed coefficients in each sub-band are set to zero and the approximate coefficients are unchanged, which is equivalent to effectively separating the fundamental frequency signal and the noise contained in the CMF output signal.

\section{B. Phase difference measurement based on EKF}

When CMF is used to stabilize single-phase flow measurements, the output signals are generally considered to be sinusoidal signals of constant amplitude and frequency. However, harmonic noise and random noise are also found in actual output signals. The discrete form of CMF signals detected by electromagnetic sensors after processing with the Mallat algorithm can be expressed as

$$
\begin{gathered}
z_{k}=\hat{z}_{k}+v_{k} \\
\hat{z}_{k}=A_{k} \sin \left(k \omega_{k}+\theta_{k}\right) \\
v_{k} \rightarrow N\left(0, \sigma^{2}\right)
\end{gathered}
$$

where $k(k=1,2, \ldots n)$ is a sampling instance; $z_{k}$ is the instantaneous value of the output signal; $\hat{z}_{k}$ is the estimated value of the output signal; $A_{k}$ is the signal amplitude; $\theta_{k}$ is the phase; the angular frequency is $\omega_{k}=2 \pi f_{k} / f_{s}, f_{s}$ is the sampling frequency; and $v_{k}$ is a small amount of interference signal after filtering, taking the white noise with zero mean.

The amplitude, phase, and frequency of the signal are used to establish the state vector, as shown below:

$$
x_{k}=\left[\begin{array}{c}
x_{1_{k}} \\
x_{2_{k}} \\
x_{3_{k}}
\end{array}\right]=\left[\begin{array}{c}
A_{k} \\
k \omega_{k}+\theta_{k} \\
f_{k}
\end{array}\right]
$$

where $x_{1_{k}}$ is the amplitude condition; $x_{2_{k}}$ is the amount of phase state; and $x_{3_{k}}$ is the frequency condition.

For stable single-phase flow measurements, the CMF's output signal state transition equation is described as follows. In the absence of control inputs, a discrete-time nonlinear process of a CMF's output signal for stable single-phase flow measurements can be expressed by the following state-space description:

$$
\begin{gathered}
x_{k}=\left[\begin{array}{ccc}
1 & 0 & 0 \\
0 & 1 & 2 \pi / f_{s} \\
0 & 0 & 1
\end{array}\right] x_{k-1}+w_{k}=F x_{k-1}+w_{k} \\
z_{k}=h\left(x_{k}\right)+v_{k}=x_{1_{k}} \sin \left(x_{2_{k}}\right)+v_{k}
\end{gathered}
$$

where $x_{k+1}$ is the process state; $z_{k}$ is the measurable output; $F$ is the system transition matrix; $h\left(x_{k}\right)$ is the measurement function; $w_{k}$ is the process noise, and $v_{k}$ is the measurement noise, represented as $w_{k} \rightarrow N\left(0, Q_{k}\right)$ and $v_{k} \rightarrow N\left(0, R_{k}\right)$.

A non-linear relationship exists between the measurements; or, the process itself is non-linear in nature. For this reason, EKF is preferable for use for dynamic state estimation. The EKF can be applied to nonlinear systems by first-order truncation of a Taylor series expansion of the nonlinear functions.

In a Taylor series expansion of the nonlinear function, the primary term coefficients are approximated so that each estimate can be performed under the linear model. The EKF technique is a two-stage recursive process of prediction and updating following an initialization step [16], [17]. The EKF procedure is summarized in the following algorithm.

Step 1: Filter initialization

The initial state estimate and its associated variance are $\hat{x}_{0}$ and $P_{0}$, respectively. 
Step 2: Prediction based on previous estimated states

Use the measured and estimated states at the previous step to predict the state and covariance matrix using the following relationships.

$$
\begin{gathered}
\hat{x}_{k, k-1}=f\left(\hat{x}_{k-1}\right) \\
P_{k, k-1}=F P_{k-1} F^{T}+Q_{k-1}
\end{gathered}
$$

where $\hat{x}_{k, k-1}$ is the a priori state estimate; $P_{k, k-1}$ is the a priori covariance matrix; $P_{k-1}$ is the a posteriori covariance matrix; and the system transition matrix is the constant matrix

$$
F=\left[\begin{array}{ccc}
1 & 0 & 0 \\
0 & 1 & 2 \pi / f_{s} \\
0 & 0 & 1
\end{array}\right]
$$

Step 3: Update step

The measured output equation can be linearized as

$$
z_{k}=H_{k} x_{k}+\tilde{v}_{k}
$$

where

$$
\begin{gathered}
H_{k}=\left.\frac{\partial h(x)}{\partial x}\right|_{x=\hat{x}_{k, k-1}}=\left[\begin{array}{c}
\sin \left(\hat{x}_{2(k, k-1)}\right) \\
\hat{x}_{1(k, k-1)} \cos \left(\hat{x}_{2(k, k-1)}\right) \\
2 \pi / f_{s} \hat{x}_{1(k, k-1)} \cos \left(\hat{x}_{2(k, k-1)}\right)
\end{array}\right] \\
\tilde{v}_{k}=v_{k}+h\left(x_{k}\right)-H_{k} x_{k}
\end{gathered}
$$

The new state updated through EKF can be written as Kalman Gain:

$$
G_{k, k-1}=P_{k, k-1} H_{k}^{T}\left(H_{k} P_{k, k-1} H_{k}{ }^{T}+R_{k}\right)^{-1}
$$

State matrix:

$$
\hat{x}_{k}=\hat{x}_{k, k-1}+G_{k}\left(z_{k}-h\left(\hat{x}_{k, k-1}\right)\right)
$$

Covariance matrix:

$$
P_{k}=\left(I-G_{k} H_{k}\right) P_{k, k-1}
$$

From the above steps, the state estimation value $\hat{x}_{k}$ can be obtained, thereby obtaining the real-time amplitude, phase, and frequency of the two output signals and, finally, calculating the phase difference $\Delta \theta$.

\section{SIMULATION AND EXPERIMENTAL RESULTS}

Simulations and actual experiments were conducted to verify the proposed method.

\section{A. Simulation of noise reduction}

In order to validate the performance of the Mallat algorithm applied in the CMF, Mallat algorithm simulations were conducted first. In these simulations, the signal frequency was set to $104.32 \mathrm{~Hz}$ and the sampling frequency to $10 \mathrm{kHz}$. The signal also contained noise, including double harmonics, higher harmonics, and white noise with an amplitude of $0.1 \mathrm{~V}$. The signal-to-noise ratio (SNR) of the simulated signal was $19.6 \mathrm{~dB}$.

The Db40 wavelet function was selected, and the CMF signal was decomposed into five layers. The de-noised signal was reconstructed according to the low frequency coefficients of the different frequency bands. Fig.1. and Fig.2 .represent the time domain waveform and the frequency spectrum of the filtered signal, respectively. It can be seen from Fig.1. that the method achieves stability after approximately 0.05 seconds. The filtered signal retains the characteristics of the original signal, and the SNR equals $32.7 \mathrm{~dB}$. As shown in Fig.2., the Mallat algorithm provides good filtering effects for both harmonics and random noise.

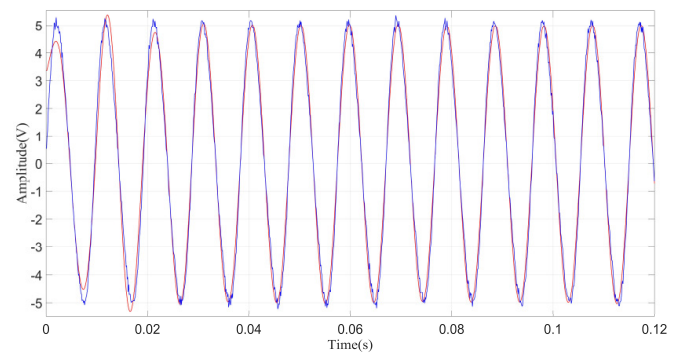

Fig.1. Time domain waveforms of the original signal (blue) and the signal filtered by the Mallat algorithm (red).

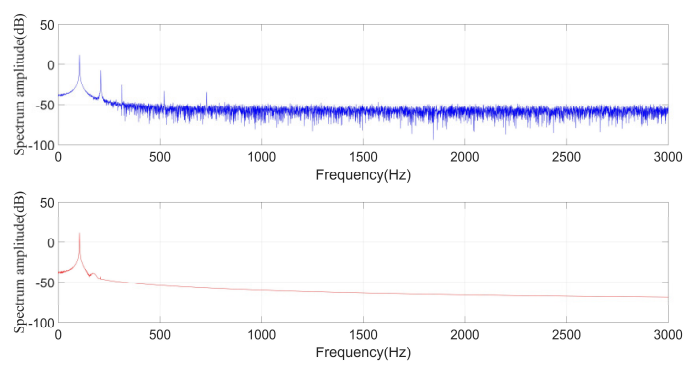

Fig.2. Spectrograms of original signal (blue) and signal filtered by the Mallat algorithm(red).

\section{B. Simulation of phase difference measurement}

In order to validate the effectiveness of the proposed algorithm, computer simulations were conducted. The properties of the proposed phase difference measurement method were analyzed by the Hilbert transform and the discrete time Fourier transform with negative frequency contribution (DTFT in [10] for short) for comparison. Hilbert transform has the advantage of being able to measure the phase difference independent of signal frequency. The phase difference measurement results are presented in Fig.3., which shows that the proposed method can track the phase difference of the signal well in the vicinity of $0.05 \mathrm{~s}$ (the black 
dotted line indicates the error limit of $\pm 0.2 \%$ ). Fig.4. shows the relative errors in phase difference between sampled points 2000 to 3000. As shown in Fig.4., the proposed method has better ability to suppress random noise and harmonics compared with the Hilbert transform and DTFT in [10].

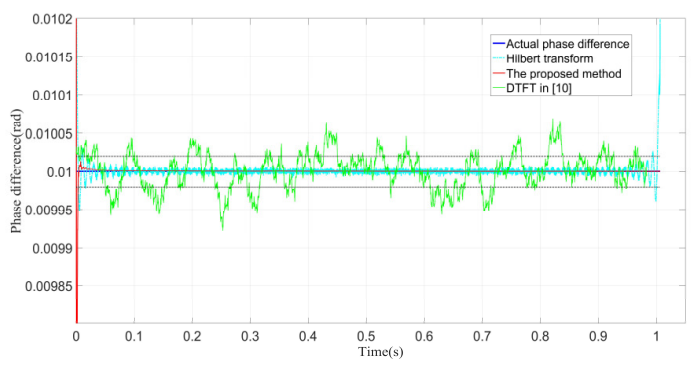

Fig.3. Comparison of phase difference measurement performance.

The mean square error (MSE) was calculated for comparison by (16), and the results are shown in Table 1.

$$
M S E=\frac{1}{N-m} \sum_{i=m}^{N}[\hat{\omega}(i)-\omega(i)]^{2}
$$

where $\omega(i)$ and $\hat{\omega}(i)$ are the actual phase difference and estimated phase difference, respectively. $m$ and $N$ are the beginning and end of the computing simulated signal, respectively, and equal 3000 and 10000.

It can be seen from Table 1. that compared with the Hilbert transform and DTFT in [10], the relative error measured by the proposed method is one order of magnitude more accurate and the MSE is two orders of magnitude more accurate, which demonstrates that the proposed method has higher precision.

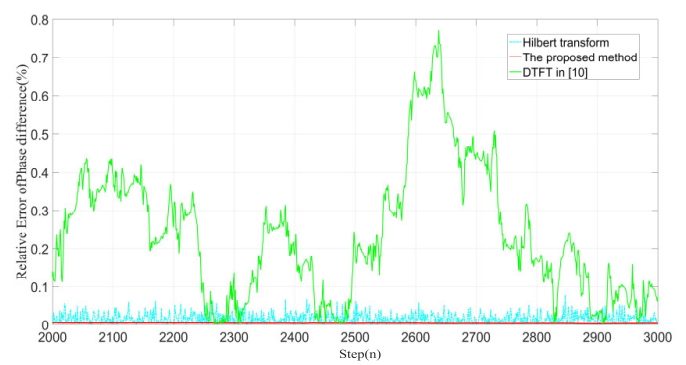

Fig.4. Relative errors in phase difference between sampled points 2000 to 3000 .

Table 1. Measurement errors under different phase differences.

\begin{tabular}{|c|c|c|c|c|c|c|}
\hline $\begin{array}{l}\text { Initial phase } \\
\text { difference } \\
(\text { rad) }\end{array}$ & \multicolumn{4}{|l|}{ Phase difference relative error (\%) } & \multicolumn{3}{l|}{ Phase difference MSE } \\
\cline { 2 - 7 } & $\begin{array}{l}\text { DTFT in } \\
{[\mathbf{1 0 ]}}\end{array}$ & $\begin{array}{l}\text { Hilbert } \\
\text { transform }\end{array}$ & $\begin{array}{l}\text { Proposed } \\
\text { method }\end{array}$ & $\begin{array}{l}\text { DTFT } \\
\left(\times \mathbf{1 0}^{-10}\right)\end{array}$ & $\begin{array}{l}\text { Hilbert } \\
\text { transform } \\
\left(\times \mathbf{1 0}^{-10}\right)\end{array}$ & $\begin{array}{l}\text { Proposed } \\
\text { method } \\
\left(\times \mathbf{1 0}^{-12}\right)\end{array}$ \\
\hline 0.0070 & 0.2718 & 0.0377 & 0.0024 & 11.859 & 0.4869 & 0.0426 \\
\hline 0.0100 & 0.2365 & 0.0384 & 0.0019 & 11.543 & 1.0045 & 0.0407 \\
\hline 0.0300 & 0.0745 & 0.0381 & 0.0014 & 11.269 & 9.4741 & 0.2044 \\
\hline 0.0500 & 0.0416 & 0.0376 & 0.0051 & 10.3994 & 2.6775 & 1.1921 \\
\hline 0.0700 & 0.0280 & 0.0385 & 0.0029 & 9.0594 & 5.4869 & 1.5863 \\
\hline
\end{tabular}

Table 2. Estimated time delays and relative errors under different flow rates.

\begin{tabular}{|c|c|c|c|c|c|c|c|}
\hline \multirow{2}{*}{$\begin{array}{c}\text { Mass } \\
\text { flow rate } \\
(\mathbf{t} / \mathbf{h})\end{array}$} & $\begin{array}{c}\text { Theoretical } \\
\text { time delay } \\
(\boldsymbol{\mu s})\end{array}$ & \multicolumn{3}{|c|}{ Mean time delay $(\boldsymbol{\mu s )}$} & \multicolumn{2}{c|}{ Relative error of time delay (\%) } \\
\cline { 3 - 8 } & & $\begin{array}{c}\text { DTFT } \\
\text { in [10] }\end{array}$ & $\begin{array}{c}\text { Hilbert } \\
\text { method }\end{array}$ & $\begin{array}{c}\text { Proposed } \\
\text { method }\end{array}$ & $\begin{array}{c}\text { DTFT in } \\
{[\mathbf{1 0}]}\end{array}$ & $\begin{array}{c}\text { Hilbert } \\
\text { method }\end{array}$ & $\begin{array}{c}\text { Proposed } \\
\text { method }\end{array}$ \\
\hline 2.4 & 3.8182 & 3.7889 & 3.8239 & 3.8172 & -0.767 & 0.149 & -0.026 \\
\hline 10.0 & 16.1043 & 15.9803 & 16.1280 & 16.1153 & -0.770 & 0.147 & 0.068 \\
\hline 20.0 & 30.2032 & 29.9703 & 30.2478 & 30.2139 & -0.771 & 0.148 & 0.035 \\
\hline 24.0 & 36.1008 & 35.8220 & 36.1551 & 36.1161 & -0.772 & 0.150 & 0.042 \\
\hline
\end{tabular}




\section{Experimental results}

Experimental data under different flow rates were gathered through a BHDN40-020-1 CMF with a BHSH1001-2 transmitter and sensor frequency of $96 \mathrm{~Hz}$. For different flowrates, the theoretical time delays and those estimated by the Hilbert transform, DTFT in [10] and proposed method were calculated (Table 2.). As shown in Table 2., the relative errors in the time delays estimated by the proposed method were smaller than those of the other two methods, which demonstrates the superior performance of the former. The reason why the relative error of the experimental data of the two algorithms is greater than the simulation error is that the experimental data contains a small amount of harmonic noise, and the proposed algorithm has a certain error in the random noise estimation.

\section{CONCLUSIONS}

This paper studies the possible applications of EKF in the signal processing of Coriolis mass flowmeters. The SGA and DTFT algorithms are dependent on frequency when used to calculate the phase difference. The Hilbert transformation method does not need to calculate frequency first to calculate phase differences, but the calculation accuracy is affected by noise. To solve the aforementioned problems, a novel method based on EKF was proposed for obtaining phase differences in CMF signals. First, the Mallat algorithm was used to reduce the noise contained in CMF output signals. Second, a state-space model of CMF output signal was established for stable single-phase flow, which effectively represents the filtered output signal. Third, quadratic error can be avoided because the frequency and the phase difference are calculated by EKF simultaneously and independently. Simulations and experiments were used to demonstrate the effectiveness and superior performance of the proposed method. While the concept has been preliminarily validated, the experiments were limited to stable single-phase flow scenarios. Therefore, it is necessary to conduct further research in applied environments. For future goals, further performance enhancements will be obtained by using an improved EKF.

\section{ACKNOWLEDGMENT}

This study was supported by the Innovative Research Team in Beihang University, National Natural Science Foundation of China, under grant number 61421063; National Natural Science Foundation of China under grant number 61873021.

\section{REFERENCES}

[1] Shanmugavalli, M., Umapathy, M., Uma, G. (2010). Smart Coriolis mass flowmeter. Measurement, 43 (4), 549-555.

[2] Enz, S. (2010). Effect of asymmetric actuator and detector position on Coriolis flowmeter and measured phase shift. Flow Measurement \& Instrumentation, 21 (4), 497-503.

[3] Romano, P. (1990). Coriolis mass flow rate meter having a substantially increased noise immunity. United States Patent US4934196.
[4] Xu, K., Ni, W. (2001). A new signal processing method for Coriolis mass flowmeter. Acta Metrologica Sinica, 22 (4), 245-257.

[5] Jacobsen, E., Lyons, R. (2003). The sliding DFT. IEEE Signal Processing Magazine, 20 (2), 74-80.

[6] Yang, J., Ke, G., Jia, L. (2006). DFT in the research of phase difference measurement in Coriolis mass flow meter. Chinese Journal of Sensors \& Actuators, 19 (6), 2654-2657.

[7] Zhu, Z.H., Xu, K.J., Yang, S.L., Li, Y., Zhu, Y.Q. (2010). Implementation and test of signal processing method of Coriolis mass flow sensor for time-varying signal model. Acta Metrologica Sinica, 31 (4), 325-329.

[8] Xu, K.-J, Ni, W. (2005). A lattice notch filter based signal processing method for Coriolis mass flowmeter. Acta Metrologica Sinica, 26 (1), 49-52.

[9] Xu, K.-J, Xu,W.-F. (2007). A signal processing method based on AFF and SGA for Coriolis mass flowmeters. Acta Metrologica Sinica, 28 (1), 48-51.

[10] Tu, Y.-Q., Zhang, H. (2008). Method for CMF signal processing based on the recursive DTFT algorithm with negative frequency contribution. IEEE Transactions on Instrumentation \& Measurement, 57 (11), 2647-2654.

[11] Vucijak, N.M., Saranovac, L.V. (2010). A simple algorithm for the estimation of phase difference between two sinusoidal voltages. IEEE Transactions on Instrumentation \& Measurement, 59 (12), 3152-3158.

[12] Yang, H., Tu, Y., Zhang, H., Peng, Y. (2012). Phase difference measuring method based on SVD and Hilbert transform for Coriolis mass flowmeter. Chinese Journal of Scientific Instrument, 33 (9), 2101-2107.

[13] Liu, W.L., Zhao, L., Wang, K.Y., Feng, Z.H., Long, Q. (2013). Signal processing for Coriolis mass flowmeter based on Hilbert transform. Acta Metrologica Sinica, 34 (5), 446-451.

[14] Tu, Y., Yang, H., Zhang, H., Liu, X. (2014) . CMF signal processing method based on feedback corrected ANF and Hilbert transformation. Measurement Science Review, 14 (1), 41-47.

[15] Mallat, S.G. (1989). Multi-frequency channel decomposition of images and wavelet models . IEEE Transactions on Acoustics, Speech and Signal Processing, 37 (12), 2071-2110 .

[16] Dash, P.K., Jena, R.K., Panda, G., Routray, A. (2000). An extended complex Kalman filter for frequency measurement of distorted signals. IEEE Transactions on Instrumentation \& Measurement, 49 (4), 746-753.

[17] Routray, A., Pradhan, A.K., Rao, K.P. (2002). A novel Kalman filter for frequency estimation of distorted signals in power systems. IEEE Transactions on Instrumentation \& Measurement, 51 (3), 469-479.

Received August 16, 2018 Accepted February 07, 2019 